\title{
PHYLLOBOTHRIUM PASTINACAE N. SP. (CESTODA, TETRAPHYLLIDEA, PHYLLOBOTHRIIDAE PARASITE DE DASYATIS PASTINACA (LINNAEUS, 1758)
}

\author{
F. MOKHTAR-MAAMOURI ET Z. ZAMALI*
}

RÉSUMÉ. Nous décrivons un nouveau parasite de l'intestin spiral de Dasyatis pastinaca du Golfe de Tunis, Phyllobothrium pastinacae n. sp. (Tetraphyllidea, Phyllobothriidae).

Cette espèce de Cestode se distingue par :

- un scolex divisé en 4 bothridies bilobées, à bord frisé, portant une rangée de loculis,

- une anatomie sans champ testiculaire postvaginal,

— un atrium génital latéral s'ouvrant toujours dans le 1/4 postérieur du segment,

- environ 100 testicules.

A new Cestode Phyllobothrium pastinacae sp. n. described from the spiral valve of Dasyatis pastinaca taken from Tunis gulf.

SUMMARY. Distinguishing characteristics include:

- scolece divided into four distincty curled bilobed bothridia with marginal loculi,

— anatomy without post vaginal testes,

- lateral genital atrium always in posterior $1 / 4$ of segments,

- about 100 testes.

Au cours d'une étude sur les Plathelminthes parasites des Sélaciens du Golfe de Tunis nous avons disséqué 42 Dasyatis pastinaca dont 40 étaient parasités par différents Cestodes déjà connus (Euzet, 1959; Williams, 1968). Cependant, dans 15 de ces Dasyatidae nous avons récolté 30 Cestodes du genre Phyllobothrium Van Beneden, 1849 qui se distinguent de toutes les espèces décrites jusqu'à présent.

Nous donnons dans ce travail la description de ce nouveau Cestode que nous nommons Phyllobothrium pastinacae n. sp.

\footnotetext{
* Laboratoire de Zoologie-Biologie animale, Faculté des Sciences, Campus Universitaire, Belvédère, Tunis, Tunisie.

Accepté le 29 janvier r98r.
} 


\section{Matériel et méthodes}

Les exemplaires de Dasyatis pastinaca utilisés pour cette étude proviennent de la pêche côtière du Golfe de Tunis.

Les vers prélevés dans la moitié antérieure de l'intestin spiral de l'hôte sont fixés au Bouin-Hollande ou au Carnoy. Ils sont par la suite colorés au Carmin aluné de Grenacher.

L'étude du Cestode est faite d'après 10 préparations " in toto " montées au baume du canada. La morphologie du scolex et l'anatomie des segments sont dessinées à la chambre claire.

\section{Description}

Holotype et Paratype déposés au Muséum national d'Histoire naturelle, Paris, numéros d'enregistrement : MNHN90HB148 c VII, MNHN90HB149 c VII, MNHN $90 \mathrm{HB} 15$ oc VII

Les spécimens étudiés mesurent de $65 \mathrm{~mm}$ à $200 \mathrm{~mm}$ de longueur. La largeur varie de 0,4 , à la base du scolex, à environ $2 \mathrm{~mm}$ dans les segments en bout de chaîne.

Le scolex ( fig. 1 et 2), de forme variable selon l'état de contraction du Cestode, a une largeur maximale de $0,7 \mathrm{~mm}$. Il porte 4 bothridies bilobées de 1,8 à 2,2 $\mathrm{mm}$ de longueur et 0,4 à $0,6 \mathrm{~mm}$ de largeur. Ces bothridies, souvent contractées et repliées (fig. 1), sont bordées d'une rangée de loculis. Étalées elles montrent des bords ondulés (fig. 2) avec des loculis plus longs que larges (environ $80 \mu \mathrm{m}$ de long et $50 \mu \mathrm{m}$ de large).

Les ventouses accessoires, de petite taille (environ $80 \mu \mathrm{m}$ de diamètre) se distinguent difficilement à cause des nombreux replis des bords des bothridies.

Au pédoncule céphalique ( 3 à $5 \mathrm{~mm}$ de long et 0,3 à $0,9 \mathrm{~mm}$ de large) succède un cou ( 3 à $10 \mathrm{~mm}$ de long et 0,4 à $1 \mathrm{~mm}$ de large) simulant une segmentation.

Le strobile est formé de 250 à plus de 330 segments craspédotes. Ces derniers, d'abord plus larges que longs (1,4 mm de large et $0,2 \mathrm{~mm}$ de long) s'allongent progressivement et, dans les segments matures, deviennent presque aussi longs que larges (1,2 $\mathrm{mm}$ de long et 1,1 mm de large) puis nettement plus longs que larges. Les segments gravides libres ou proglottis ont généralement $3,2 \mathrm{~mm}$ de long et $1,9 \mathrm{~mm}$ de large.

Phyllobothrium pastinacae semble être euapolytique. En effet, les plus grands exemplaires rencontrés n'avaient pas de segments gravides en bout de chaîne.

Anatomie du segment ( $f$ ig 3 et 4 )

Le pore génital alterne irrégulièrement et s'ouvre toujours au $1 / 4$ postérieur du segment. 


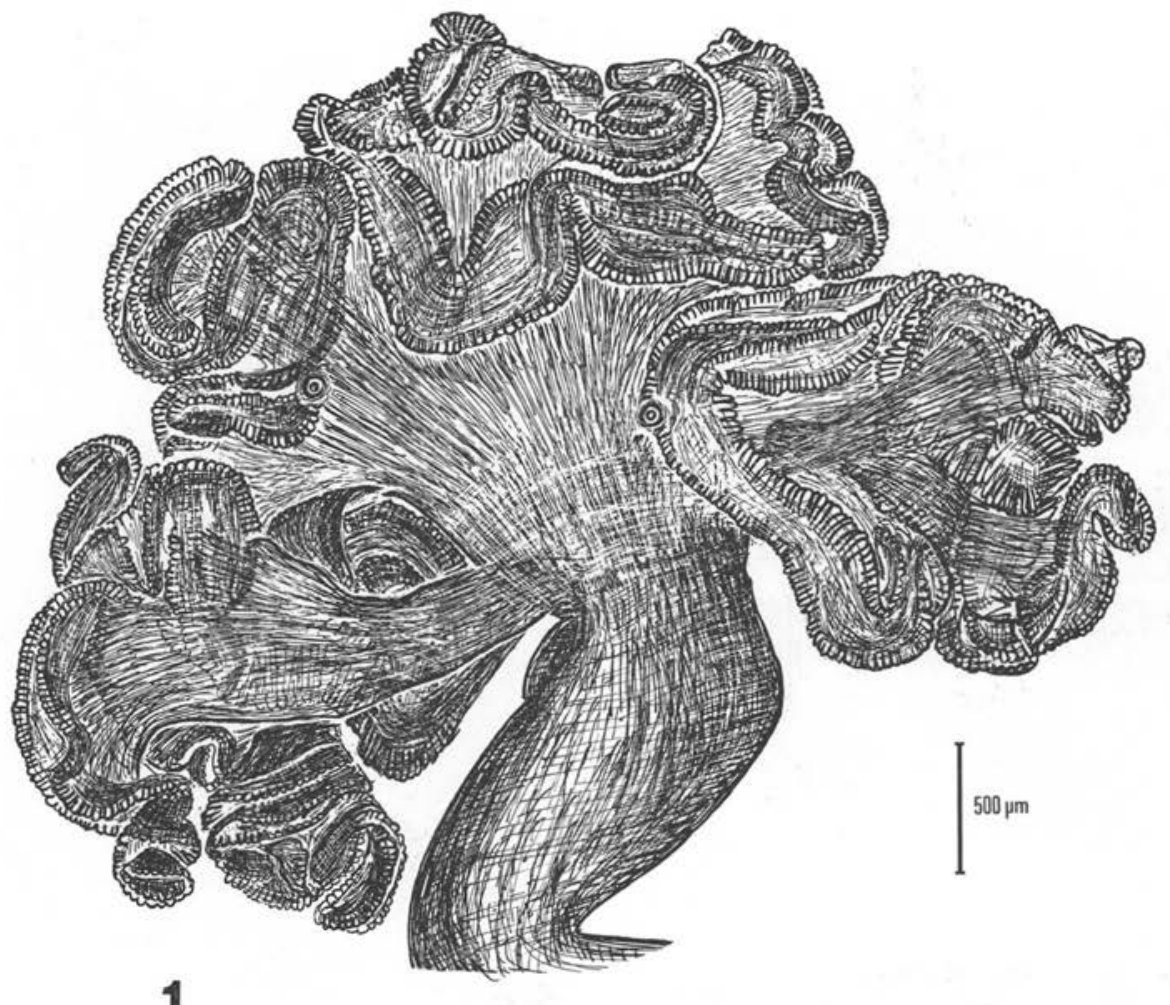

FIG. 1. - Scolex in toto légèrement aplati, montrant la ventouse accessoire de deux bothridies.

APPAREIL GÉNItAL MALE :

Nous avons compté 86 à 120 petits testicules sans champ testiculaire postvaginal.

Le canal déférent forme un peloton lâche en avant de la poche du cirre. Cette dernière mesure de 0,18 à $0,28 \mathrm{~mm}$ de large et de 0,27 à $0,37 \mathrm{~mm}$ de long. Le cirre épineux est élargi vers la base.

\section{Appareil génital femelle :}

L'ovaire occupe, environ, le $1 / 4$ postérieur du segment.

Le vagin sinueux, élargi en avant du pont ovarien en un réceptacle séminal, débouche dans l'atrium génital antérieurement à la poche du cirre.

Les vitellogènes nombreux forment deux bandes latérales. Chaque bande s'étend, dorsalement et ventralement, sur environ le 1/3 du segment. Dans les segments matures ces vitellogènes recouvrent en grande partie les testicules. 

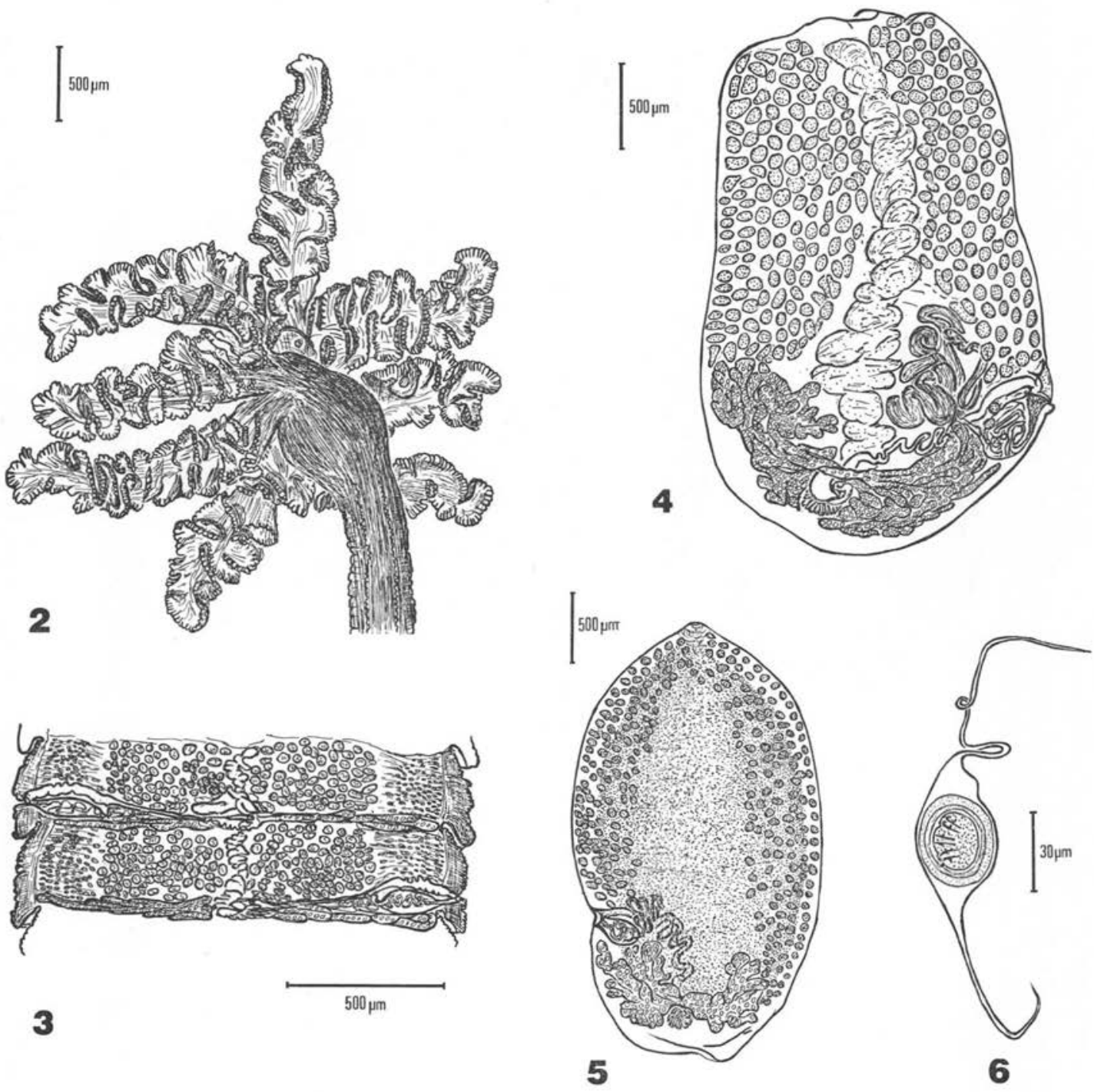

FIg. 2. - Scolex d'après un individu fixé en place dans la valvule spirale.

FIg. 3. - Anatomie d'un segment sexué.

FIG. 4. - Proglottis venant de se séparer du strobile. L'utérus médian est formé, le développement des glandes vitellogènes cache les testicules regressés.

Fig. 5. - Proglottis détaché à utérus gravide.

FIG. 6. - Euf embryonné avec expansions polaires filiformes. 
Les vitelloductes médians, situés dans la région postérieure du segment, se rejoignent sur la ligne médiane légèrement en avant du pont ovarien.

Dans les proglottis, l'utérus sacciforme occupe le 1/3 médian du segment. Il est bourré de petits œufs généralement à différents stades de l'ontogénèse. (fig 5).

Les œufs ont la forme d'un fuseau de $30 \mu \mathrm{m}$ de large et environ $60 \mu \mathrm{m}$ de long prolongé à chaque pôle par un filament mince de 80 à $120 \mu \mathrm{m}$ de longueur. L'embryon héxacanthe mesure $17 \mu \mathrm{m}$ de long et $20 \mu \mathrm{m}$ de large. (fig 6 ).

\section{Discussion}

L'anatomie de Phyllobothrium pastinacae rappelle celle de $P$. centrurum. Cependant les deux Cestodes se distinguent nettement par :

- la taille (plus de 60 à $200 \mathrm{~mm}$ au lieu de 20 à $25 \mathrm{~mm}$ ),

- la morphologie des bothridies,

- la taille et le nombre des loculis,

- le nombre des testicules (en moyenne 100 au lieu de 46).

Par l'aspect de son scolex P. pastinacae est comparable à Bothriocephalus echeneis décrit par Leuckart en 1920 (in Southwell, 1925) et à $P$. lactuca Van Beneden, 1850 redécrit par Euzet (1959). Mais chez les deux espèces citées les bords des bothridies ne sont pas loculés. D'ailleurs $P$. pastinacae se distingue aussi par l'anatomie des segments sans champ testiculaire postvaginal.

Le scolex de l'espèce que nous décrivons se rapproche de celui de $P$. tumidum donné par Tseng Shen (1933). Cependant, la présence d'un champ testiculaire postvaginal chez $P$. tumidum ainsi que la position de la poche du cirre et le nombre de testicules (300 au lieu de 86 à 120) ne nous permettent pas de les confondre.

L'anatomie du segment de $P$. pastinacae et la forme de l'œuf sont semblables à celles de $P$. gracile Wedl, 1855 . Toutefois les deux Cestodes sont nettement reconnaissables à leur scolex.

Nos spécimens représentent donc les seuls Phyllobothrium de Dasyatidae avec un scolex formé de 4 bothridies bilobées bordées d'une rangée de loculis et une anatomie sans champ testiculaire postvaginal.

\section{BIBLIOGRAPHIE}

Euzet L. : Recherches sur les Cestodes Tétraphyllides des Sélaciens des Côtes de France. Thèse, Fac. Sc. Montpellier, 1959, 263 p.

Southwell T. : A monograph of the Tetraphyllidea with notes on related Cestodes. Mem. Liverpool School. Trop. Med. New Series, 1925, 2, I-368.

Tseng Shen : Study on some Cestodes from fishes. J. Sc. Nat. Uni. of Shantung Tsingtao, 1933, 2, I-2I.

Williams H. H. : The taxonomy, ecology and host-specificity of some Phyllobothriidae (Cestoda : Tetraphyllidea), a critical revision of Phyllobothrium Beneden, 1849 and comments on some allied genera. Phil. Trans. Roy. Soc. London, 1968, Ser. B, 253, $231-307$. 